\title{
Interfaces, Strings, and a Soft Mode in the Square Lattice Quantum Dimer Model
}

\author{
D. Banerjee ${ }^{1}$, M. Bögli ${ }^{1}$, C. P. Hofmann ${ }^{2}$, F.-J. Jiang ${ }^{3}$, P. Widmer ${ }^{1}$, and U.-J. Wiese ${ }^{1}$ \\ ${ }^{1}$ Albert Einstein Center, Institute for Theoretical Physics, Bern University, Switzerland \\ ${ }^{2}$ Facultad de Ciencias, Universidad de Colima, Bernal Diaz del Castillo 340, Colima C.P. 28045, Mexico \\ 3 Department of Physics, National Taiwan Normal University 88, Sec. 4, Ting-Chou Rd., Taipei 116, Taiwan
}

\begin{abstract}
The quantum dimer model on the square lattice is equivalent to a $U(1)$ gauge theory. Quantum Monte Carlo calculations reveal that, for values of the Rokhsar-Kivelson (RK) coupling $\lambda<1$, the theory exists in a confining columnar phase. The interfaces separating distinct columnar phases display plaquette order, which, however, is not realized as a bulk phase. Static "electric" charges are confined by flux tubes that consist of multiple strands, each carrying a fractionalized flux $\frac{1}{4}$. A soft pseudo-Goldstone mode emerges around $\lambda \approx 0$, long before one reaches the RK point at $\lambda=1$.
\end{abstract}

Quantum dimer models [1 implement Anderson's ideas of resonating valence bonds [2] as a potential route towards understanding high-temperature superconductivity. A dimer connecting neighboring lattice sites represents a singlet-pair of two spins $\frac{1}{2}$. In the undoped dimer model each lattice site is touched by exactly one dimer, thus modeling a system in which each spin participates in a singlet pair. A lot of progress has been made on unraveling the phase structure of classical and quantum dimer models for various lattice geometries [3 14]. Despite the fact that they do not suffer from the notorious sign problem, quantum Monte Carlo calculations for quantum dimer models are rather limited. For the square lattice, Green's function Monte Carlo calculations [15. have so far led to inconclusive results. While 16 found evidence for a phase transition between the columnar and the plaquette phase (cf. Fig.1a,b) near $\lambda \approx 0.6$, [17] concluded that for $\lambda \gtrsim 0$ there might be a mixed phase that shares features of both columnar and plaquette phases.

The square lattice quantum dimer model is closely related to the $(2+1)$-d $U(1)$ quantum link model 18 20, a lattice gauge theory with a 2-dimensional spin $\frac{1}{2}$ Hilbert space per link, which has also been investigated in the context of spin liquids 21, 22. In fact, both models share the same Hamiltonian, but realize the Gauss law in two different ways. While the quantum link model has no background charges in its ground state, the quantum dimer model operates in a staggered background of charges \pm 1 , which ensures that each lattice site is touched by exactly one dimer. Quantum link models [20, 23, 24 provide an alternative non-perturbative regularization of gauge theories in particle physics. In the quantum link formulation of 4-d Quantum Chromodynamics, the confining gluon field emerges by dimensional reduction from a deconfined Coulomb phase of a $(4+1)$-d $S U(3)$ quantum link model, while chiral quarks arise naturally as domain wall fermions located at the two 4-d sides of a $(4+1)$-d slab 23. Thanks to their finite-dimensional link Hilbert space, quantum link models are ideally suited for the constructions of atomic quantum simulators 25 for Abelian [26-30] and non-Abelian gauge theories [31 33. using ultracold atoms in an optical lattice. a)
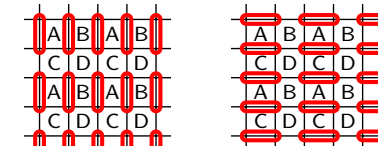

b)
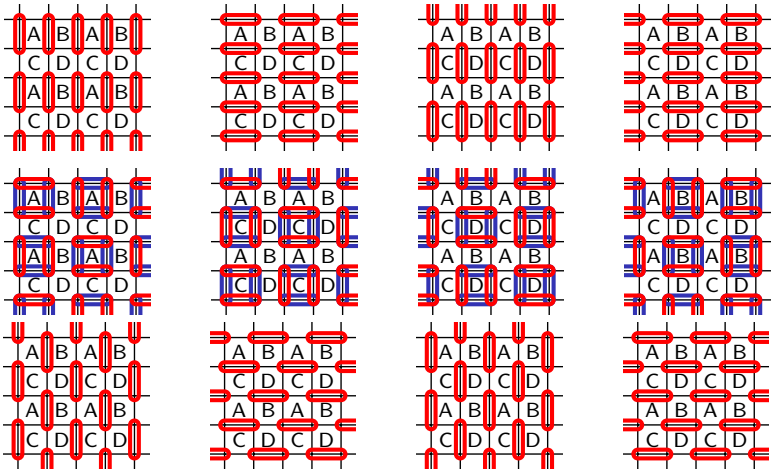

FIG. 1. [Color online] a) Columnar, b) plaquette, and c) staggered order, shown with four dual sublattices $A, B, C, D$.

Recently, we have developed a very efficient cluster algorithm to simulate the $(2+1)$-d $U(1)$ quantum link model, which has allowed us to study its confining dynamics 34. In particular, there are two distinct confining phases (analogous to columnar and plaquette phases in the quantum dimer model) with different discrete symmetry breaking patterns, separated by a weak first order phase transition that mimics several features of deconfined quantum critical points 35 37. In particular, at the phase transition a light pseudo-Goldstone boson emerges dynamically, which can, however, not be interpreted as a dual photon, because it is not exactly massless. The confining strings that connect an external charge-anti-charge pair fractionalize into mutually repelling strands, each carrying fractional flux $\frac{1}{2}$. The interior of the strands consists of the bulk phase that is stable on the other side of the phase transition.

Here we apply numerical simulations to the square lattice quantum dimer model. We find that, in the columnar phase, flux strings fractionalize into strands of flux $\frac{1}{4}$, whose interior consists of plaquette phase, which, however, is not realized in the bulk. The same is true for the interfaces separating distinct columnar phases, which display complete wetting. Unlike in the quantum link model, in the quantum dimer model there is no phase transition separating two distinct confining phases. Instead the columnar phase extends all the way to the RK 


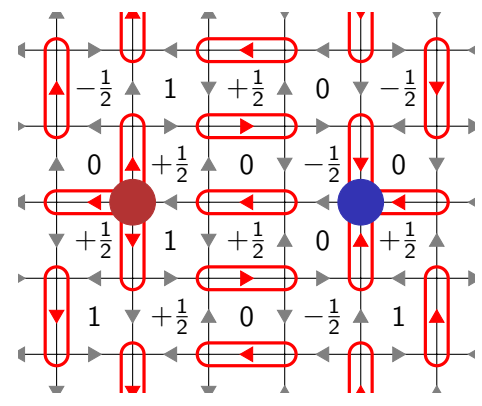

FIG. 2. [Color online] Dimer configuration with associated fluxes and dual height variables, in the presence of two external charges.

point $\lambda=1$. Remarkably, on moderate volumes the massless mode that arises at the RK point influences the entire region $0 \lesssim \lambda<1$, in which it is still relatively light.

The configurations of the quantum dimer model are characterized by variables $D_{x y} \in\{0,1\}$, indicating the presence or absence of a dimer on the link connecting the neighboring sites $x$ and $y$ on a square lattice. The Hamiltonian of the quantum dimer model is the same as the one of the $(2+1)$-d $U(1)$ quantum link model

$$
H=-J \sum_{\square}\left[U_{\square}+U_{\square}^{\dagger}-\lambda\left(U_{\square}+U_{\square}^{\dagger}\right)^{2}\right] .
$$

Here $U_{\square}=U_{w x} U_{x y} U_{z y}^{\dagger} U_{w z}^{\dagger}$ is a plaquette operator formed by quantum links $U_{x y}$ connecting nearestneighbor sites $x$ and $y$. A $U(1)$ quantum link $U_{x y}=S_{x y}^{+}$is a raising operator of electric flux $E_{x y}=S_{x y}^{3}$, constructed from a quantum spin $\frac{1}{2}$ associated with the link $x y$. As shown in Fig.2, the electric flux variables are related to the dimer variables by $E_{x y}=(-1)^{x_{1}+x_{2}}\left(D_{x y}-\frac{1}{2}\right)$. The first term in the Hamiltonian rotates a pair of parallel dimers on opposite links of a plaquette by 90 degrees. Equivalently, it flips a loop of electric flux, winding around the plaquette. It also annihilates non-flippable plaquette states, while the RK term, proportional to $\lambda$, counts flippable plaquettes. The Hamiltonian commutes with the generators $G_{x}=\sum_{i}\left(E_{x, x+\hat{i}}-E_{x-\hat{i}, x}\right)$ of infinitesimal $U(1)$ gauge transformations. In allowed configurations exactly one dimer touches each site, such that

$$
G_{x}=(-1)^{x_{1}+x_{2}} \sum_{i}\left(D_{x, x+\hat{i}}+D_{x-\hat{i}, x}\right)=(-1)^{x_{1}+x_{2}},
$$

where $\hat{i}$ is the unit-vector in the $i$-direction. The dimer covering constraint is thus equivalent to a staggered background of electric charges \pm 1 . While in the quantum link model physical states obey $G_{x}|\Psi\rangle=0$, in the quantum dimer model they obey $G_{x}|\Psi\rangle=(-1)^{x_{1}+x_{2}}|\Psi\rangle$.

Besides the $U(1)$ gauge symmetry, the model also has several global symmetries, including translations by one lattice spacing combined with charge conjugation, $\mathrm{CT}_{x}$ and $\mathrm{CT}_{y}$ (which correspond to ordinary translations of

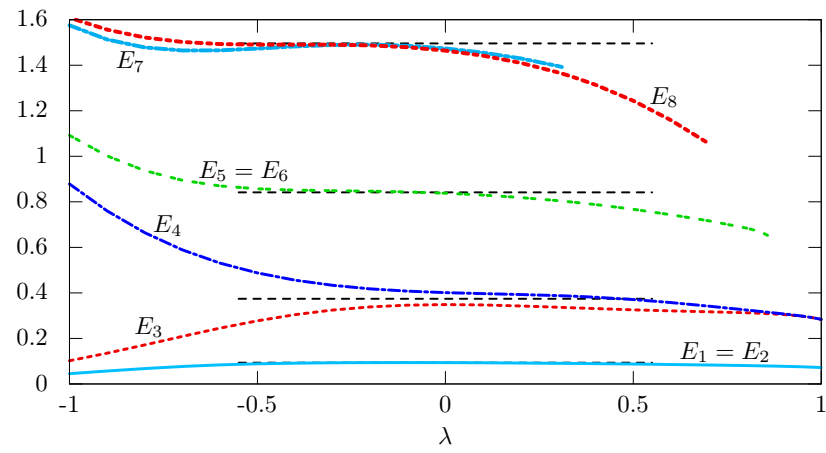

FIG. 3. [Color online] Energy spectrum on an $8^{2}$ lattice as a function of the $R K$ coupling $\lambda$.

the dimers $D_{x y}$ ), a 90 degrees rotation $\mathrm{O}$ around a lattice point, as well as a 90 degrees rotation around a plaquette center combined with charge conjugation $\mathrm{CO}^{\prime}$. Another important global symmetry is the $U(1)^{2}$ center symmetry associated with "large" gauge transformations [38. On an $L_{1} \times L_{2}$ lattice with periodic boundary conditions, there are super-selection sectors characterized by wrapping electric fluxes $E_{i}=\frac{1}{L_{i}} \sum_{x} E_{x, x+\hat{i}} \in \mathbb{Z} / 2$. They commute with the Hamiltonian, but cannot be expressed through "small" periodic gauge transformations $G_{x}$.

Following [17, we have performed exact diagonalization studies on $L_{1} \times L_{2}$ lattices with $L_{1}, L_{2} \in\{4,6,8\}$. The energies of some of the lowest states are illustrated in Fig.3. For $\lambda<1$, the ground state has quantum numbers $\left(\mathrm{CT}_{x}, \mathrm{CT}_{y}\right)=(+,+)$. The first two excited states with energy gap $E_{1}=E_{2}$ are degenerate and have quantum numbers $(+,-)$ and $(-,+)$, while the next excited state with energy gap $E_{3}$ has quantum numbers $(+,+)$. For $\lambda \lesssim-0.2$, the energy gaps $E_{1,2}, E_{3} \sim \exp \left(-\alpha L_{1} L_{2}\right)$ decrease exponentially with the volume $L_{1} L_{2}$, thus indicating the spontaneous breakdown of translation invariance that characterizes the columnar phase. For $-0.2 \lesssim \lambda \lesssim 0.8$, a $(-,-)$ state with energy $E_{4} \approx E_{3}$ almost degenerates with the $(+,+)$ state. The next exactly degenerate states with energy $E_{5}=E_{6}$ again have quantum numbers $(+,-)$ and $(-,+)$, while the states with energies $E_{7}$ and $E_{8}$ are almost degenerate and transform as $(+,+)$ and $(-,-)$. As indicated by the dashed lines in Fig.3, the energy ratios of these states are given by $E_{1,2}: E_{3,4}: E_{5,6}: E_{7,8} \approx 1: 4: 9: 16$, thus indicating an approximate rotor spectrum and possibly the transition to a different phase. However, the volumes accessible to exact diagonalization are too small to come to a definitive conclusion concerning this issue.

Green's function Monte Carlo simulations of quantum dimer models have been performed in [15-17] on systems of size up to $48^{2}$. While [16] reached the conclusion that the columnar phase turns into the plaquette phase near $\lambda \approx 0.6,17$ interpreted the data in terms of a mixed phase that shares features of the columnar and the plaquette phase. Here we apply an alternative numerical 
method on volumes up to $144^{2}$, and conclude that the columnar phase extends all the way to the RK point. First, we introduce dual height variables associated with two even $(A, D)$ and two odd $(B, C)$ dual sublattices (cf. Fig.1), $h_{\widetilde{x}}^{A, D}=0,1, h_{\widetilde{x}}^{B, C}= \pm \frac{1}{2}$, located at the dual sites $\widetilde{x}=\left(x_{1}+\frac{1}{2}, x_{2}+\frac{1}{2}\right)$. They are associated with a flux configuration $E_{x, x+\hat{1}}=\left[h_{\widetilde{x}}^{X}-h_{\widetilde{x}-\hat{2}}^{X^{\prime}}\right] \bmod 2= \pm \frac{1}{2}, E_{x, x+\hat{2}}=$ $(-1)^{x_{1}+x_{2}}\left[h_{\widetilde{x}}^{X}-h_{\widetilde{x}-\hat{1}}^{X^{\prime}}\right] \bmod 2= \pm \frac{1}{2}, X, X^{\prime} \in\{A, B, C, D\}$. The different symmetry breaking patterns are distinguished by four order parameters, $M_{X}=\sum_{\widetilde{x} \in X} s_{\widetilde{x}}^{X} h_{\widetilde{x}}^{X}$, with $s_{\widetilde{x}}^{A}=s_{\widetilde{x}}^{C}=(-1)^{\left(\widetilde{x}_{1}+\frac{1}{2}\right) / 2}\left(\widetilde{x}_{1}+\frac{1}{2}\right.$ even $), s_{\widetilde{x}}^{B}=s_{\widetilde{x}}^{D}=$ $(-1)^{\left(\widetilde{x}_{1}-\frac{1}{2}\right) / 2}\left(\widetilde{x}_{1}+\frac{1}{2}\right.$ odd $)$. The order parameters

$$
\begin{aligned}
& M_{11}=M_{A}-M_{B}-M_{C}+M_{D}=M_{1} \cos \varphi_{1}, \\
& M_{22}=M_{A}+M_{B}-M_{C}-M_{D}=M_{1} \sin \varphi_{1}, \\
& M_{12}=M_{A}-M_{B}-M_{C}-M_{D}=M_{2} \cos \varphi_{2}, \\
& M_{21}=-M_{A}+M_{B}-M_{C}-M_{D}=M_{2} \sin \varphi_{2},
\end{aligned}
$$

give rise to $\varphi=\frac{1}{2}\left(\varphi_{1}+\varphi_{2}+\frac{\pi}{4}\right)$, which transforms as

$$
\begin{aligned}
& { }^{C T_{x}} \varphi=\pi-\varphi, \quad{ }^{C T_{y}} \varphi=\frac{\pi}{2}-\varphi, \\
& { }^{O} \varphi=\frac{\pi}{4}+\varphi, \quad{ }^{C O^{\prime}} \varphi=-\frac{\pi}{4}-\varphi .
\end{aligned}
$$

It should be noted that $\pm\left(M_{A}, M_{B}, M_{C}, M_{D}\right)$ (and thus $\varphi$ and $\varphi+\pi)$ represent the same physical configuration, because shifting the height variables to $h_{\widetilde{x}}^{X}(t)^{\prime}=\left[h_{\widetilde{x}}^{X}(t)+\right.$ 1]mod2 leaves the dimer configuration unchanged. The columnar phases correspond to $\varphi=0 \bmod \frac{\pi}{4}$, while the plaquette phases correspond to $\varphi=\frac{\pi}{8} \bmod \frac{\pi}{4}$.

We have performed quantum Monte Carlo simulations with a Metropolis algorithm applied to the dual height variables. The algorithm is restricted to a fixed sector of wrapping electric fluxes (here $E_{i}=0$ ), but updates a given sector ergodically. For $\lambda<1$, at low temperatures the restriction to $E_{i}=0$ is no problem, because the ground state belongs to this sector. About three quarters of all height variable flips are forbidden because they violate the dimer covering constraint, and only a few percent of the proposed flips are accepted in the Metropolis step. Although the algorithm thus has a rather small acceptance rate, it works remarkably well and allows us to access volumes as large as $144^{2}$ and temperatures as low as $T=J / 500$. The algorithm has been used to determine the probability distribution $p\left(M_{11}, M_{22}\right)$ shown in Fig.4a,b at $\lambda=-0.5$ and 0.8 for $L_{1}=L_{2}=24 a$, which reveals an emergent approximate spontaneously broken $S O(2)$ symmetry for $\lambda \gtrsim-0.2$.

The angle $\varphi$ parametrizes the associated soft pseudoGoldstone mode. Since $\varphi$ and $\varphi+\pi$ are physically indistinguishable, only those states that are invariant against this shift belong to the physical Hilbert space. The corresponding low-energy effective theory is a $(2+1)$-d $\mathbb{R} P(1)$ model with the Euclidean Lagrangian

$$
\mathcal{L}=\frac{\rho_{t}}{2} \partial_{t} \varphi \partial_{t} \varphi+\frac{\rho}{2} \partial_{i} \varphi \partial_{i} \varphi+\kappa\left(\partial_{i} \partial_{i} \varphi\right)^{2}+\delta \cos ^{2}(4 \varphi) .
$$
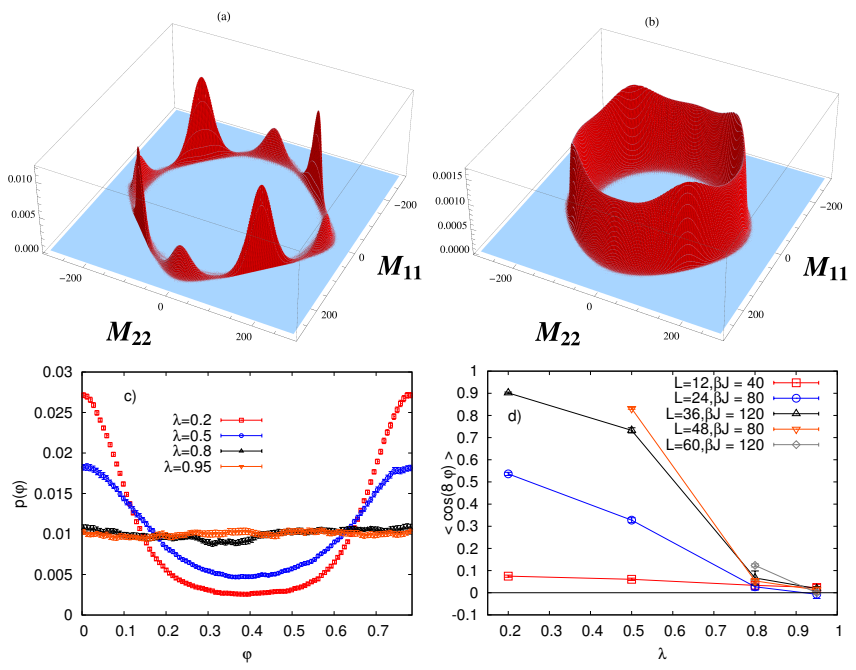

FIG. 4. [Color online] Probability distribution $p\left(M_{11}, M_{22}\right)$ : a) $\lambda=-0.5, \beta J=50$, b) $\lambda=0.8, \beta J=100, L_{1}=L_{2}=24 a$. c) Probability distribution $p(\varphi)$ and $d)\langle\cos (8 \varphi)\rangle$.

The effective theory predicts a finite-volume rotor spectrum (given simply by $E_{m}=m^{2} /\left(2 \rho_{t} L_{1} L_{2}\right)$ when assuming $\delta=0$ ) with $m=0, \pm 2, \pm 4, \ldots$. States with odd values of $m$ are excluded because they are not invariant against a shift of $\varphi$ by $\pi$. In fact, the nine states with $m=0, \pm 2, \pm 4, \pm 6, \pm 8$ have exactly the same $\left(\mathrm{CT}_{x}, \mathrm{CT}_{y}\right)$ quantum numbers as the ones obtained by exact diagonalization (cf. Fig.3). The $\delta$-term explicitly breaks the emergent $S O(2)$ symmetry to a $\mathbb{Z}(8)$ subgroup and gives rise to a small Goldstone boson mass. The value of $\delta$ can be derived from a finite-size analysis of the probability distribution $p(\varphi)$ illustrated in Fig.4c. Inspired by [39, we have determined the moment $\langle\cos (8 \varphi)\rangle=\int_{-\pi}^{\pi} d \varphi p(\varphi) \cos (8 \varphi)$ for different $\lambda$ (cf. Fig.4d). We find that $\langle\cos (8 \varphi)\rangle>0$ for $\lambda<1$, indicating that the system remains in the columnar phase all the way to the RK point. In mean field theory the most general quartic potential is given by

$$
\begin{aligned}
& V=\mu_{1} O_{1}+\mu_{2} O_{2}+\lambda_{0} O_{1} O_{2}+\sum_{i=1}^{5} \lambda_{i}\left|O_{i}\right|^{2}, \\
& O_{1}=M_{11}^{2}+M_{22}^{2}+M_{12}^{2}+M_{21}^{2}, \\
& O_{2}=M_{11} M_{12}-M_{11} M_{21}+M_{22} M_{12}+M_{22} M_{21}, \\
& O_{3}=M_{11}^{2}+M_{22}^{2}-M_{12}^{2}-M_{21}^{2}, \\
& O_{4}=M_{11} M_{12}+M_{11} M_{21}-M_{22} M_{12}+M_{22} M_{21}, \\
& O_{5}=M_{11} M_{22}+i M_{12} M_{21} .
\end{aligned}
$$

A systematic analytic analysis of the minima of $V$ in the infinitesimal neighborhood of the staggered phase (which appears for $\lambda>1$ and has $M_{A}=M_{B}=M_{C}=M_{D}=0$, cf. Fig.1c) shall be presented elsewhere.

It is natural to define the dual field $F_{\mu \nu}(x)=$ $\frac{1}{\pi} \varepsilon_{\mu \nu \rho} \partial_{\rho} \varphi(x)$. Vortices and half-vortices in the order parameter field manifest themselves as charges. The electric 
charge contained in a spatial region $\Omega$ is given by twice the vortex number

$$
Q_{\Omega}=\int_{\Omega} d^{2} x \partial_{i} F_{0 i}=\frac{1}{\pi} \int_{\partial \Omega} d \sigma_{i} \varepsilon_{i j} \partial_{j} \varphi \in \frac{\mathbb{Z}}{2} .
$$

Note that a charge 1 corresponds to a half-vortex, which is allowed because $\varphi$ and $\varphi+\pi$ are physically equivalent. The flux of $F_{\mu \nu}$ represents the conserved charges of the $U(1)^{2}$ center symmetry. At the RK point electric flux costs zero energy and condenses in the vacuum, thus giving rise to deconfinement even at zero temperature. In the effective theory, this implies that $\partial_{i} \varphi$ does not contribute to the energy, and hence that $\rho=\delta=0$ at $\lambda=1$.

Figs.5a,b illustrate the energy distribution in an interface that separates two distinct columnar phases with the columns oriented in the $y$-direction. By complete wetting, this interface splits into two interfaces enclosing an intermediate columnar phase with the columns oriented in the $x$-direction. The two interfaces separating the three columnar phases display plaquette order. While the plaquette ordered region increases as one approaches the RK point, it never becomes a stable bulk phase.

Let us also investigate configurations with two external static charges \pm 2 relative to the staggered charge background, i.e. with $G_{x}=-(-1)^{x_{1}+x_{2}}$ at two positions separated by an odd number of lattice spacings. This violation of the dimer covering constraint implies that three dimers touch each of those two sites (cf. Fig.2). The two external charges are confined by an electric flux string. Interestingly, the total flux 2 connecting the external charges \pm 2 fractionalizes into eight strands, each carrying electric flux $\frac{1}{4}$. The interior of the strands again displays plaquette order and represent interfaces separating distinct columnar phases whose columns are oriented in orthogonal directions. The energy distribution in the charge-anti-charge configuration is illustrated in Fig.5c. The energy of the string plays the role of a confining charge-anti-charge potential $V(r) \sim \sigma r$, which is shown in Fig.5d. Deep in the columnar phase, the string tension is given by $\sigma(\lambda=-0.5)=0.370(1) \mathrm{J} / \mathrm{a}$. With increasing $\lambda$, it is greatly reduced to $\sigma(\lambda=0.5)=0.057(1) \mathrm{J} / \mathrm{a}$, $\sigma(\lambda=0.8)=0.029(1) \mathrm{J} / \mathrm{a}$, and it finally vanishes at the deconfined RK point with $\sigma(\lambda=1)=0$.

In conclusion, we found that the $(2+1)$-d quantum dimer model is in a columnar phase for all values of the RK coupling $\lambda<1$, without ever going into a plaquette phase. At $\lambda=1$, it is well known that electric fluxes condense in the vacuum, thus leading to deconfinement even at zero temperature. This corresponds to the spontaneous breakdown of the $U(1)^{2}$ center symmetry. In the confining columnar phase, the string connecting two external static charges separates into distinct strands, each carrying a fractionalized flux $\frac{1}{4}$. The interior of the flux strands consists of plaquette phase which does, however, not exist as a stable bulk phase. We have observed an approximate emergent $S O(2)$ symmetry with an associ-
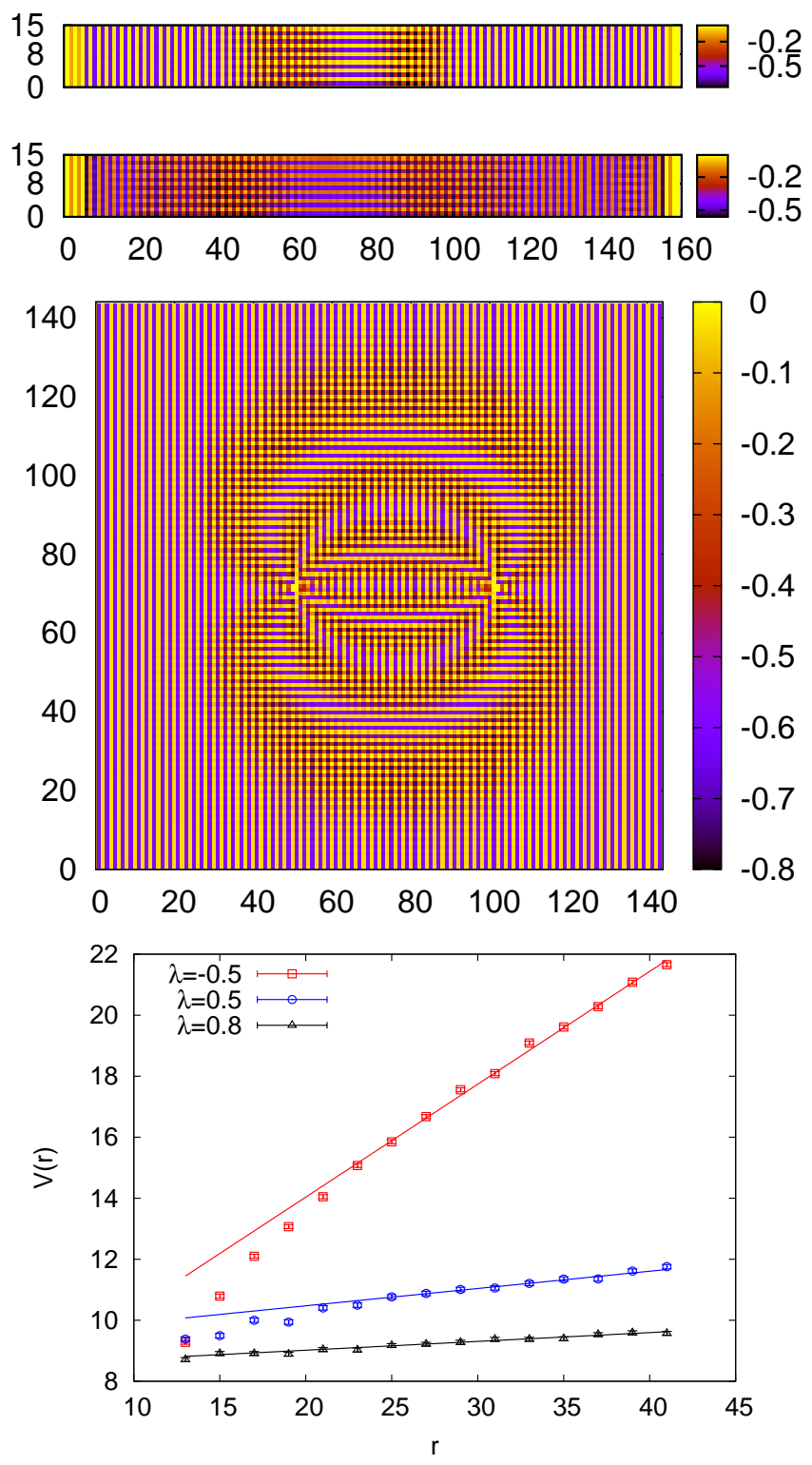

FIG. 5. [Color online] From top to bottom: Interfaces at a) $\lambda=-0.5(\beta J=100)$ and $b) \lambda=0.7(\beta J=500)$, on $a 16 \times$ 160 lattice. c) Energy density $-J\left\langle U_{\square}+U_{\square}^{\dagger}\right\rangle$ in the presence of two charges \pm 2 (separated by 49 lattice spacings) for $\lambda=$ $-0.2, \beta J=72$, on a $144^{2}$ lattice. d) Potential between two static charges \pm 2 separated by a distance $r$ along a lattice axis, for $\lambda=-0.5,0.5,0.8$.

ated pseudo-Goldstone boson. At $\lambda=1$ the Goldstone boson becomes exactly massless, and can be identified with a dual photon. Remarkably, as indicated by the rotor spectrum in Fig.3, the pseudo-Goldstone mode, which represents a soft phonon-like mode of the columnar valence bond solid, exists even far away from the RK point at $\lambda \approx 0$. It will be interesting to investigate whether this "phonon" mode persists in doped systems and whether it may be related to the formation of Cooper pairs in high- $T_{c}$ superconductors. 
UJW likes to thank the CTP at MIT, where this work was initiated, for hospitality during a sabbatical. D. B. acknowledges interesting discussions with A. Läuchli. The research leading to these results has received funding from the Schweizerischer Nationalfonds and from the European Research Council under the European Union's Seventh Framework Programme (FP7/2007-2013)/ ERC grant agreement 339220 .

[1] D. S. Rokhsar and S. A. Kivelson, Phys. Rev. Lett. 61 (1988) 2376.

[2] P. W. Anderson, Science 235 (1987) 1196.

[3] S. Sachdev, Phys. Rev. B40 (1989) 5204.

[4] L. S. Levitov, Phys. Rev. Lett. 64 (1990) 92.

[5] P. W. Leung, K. C. Chiu, and K. J. Runge, Phys. Rev. B54 (1996) 12938.

[6] R. Moessner, S. L. Sondhi, and E. Fradkin, Phys. Rev. B65 (2002) 024504.

[7] C. L. Henley, J. Phys.: Condens. Matter 16 (2004) S891.

[8] F. Alet, J. L. Jacobsen, G. Misguich, V. Pasquier, F. Mila, and M. Troyer, Phys. Rev. Lett. 94 (2005) 235702.

[9] F. Alet, Y. Ikhlef, J. L. Jacobsen, G. Misguich, and V. Pasquier, Phys. Rev. E74 (2006) 041124.

[10] D. Charrier and F. Alet, Phys. Rev. B82 (2010) 014429.

[11] J. Cano and P. Fendley, Phys. Rev. Lett. 105 (2010) 067205.

[12] A. F. Albuquerque and F. Alet, Phys. Rev. B82 (2010) 180408(R).

[13] Y. Tang, A. W. Sandvik, and C. L. Henley, Phys. Rev. B84 (2011) 174427.

[14] C. A. Lamas, A. Ralko, M. Oshikawa, D. Poilblanc, and P. Pujol, Phys. Rev. B87 (2013) 104512.

[15] O. F. Syljuasen, Phys. Rev. B71 (2005) 020401(R).

[16] O. F. Syljuasen, Phys. Rev. B73 (2006) 245105.

[17] A. Ralko, D. Poilblanc, and R. Moessner, Phys. Rev. Lett. 100 (2008) 037201.

[18] D. Horn, Phys. Lett. 100B (1981) 149.
[19] P. Orland and D. Rohrlich, Nucl. Phys. B338 (1990) 647.

[20] S. Chandrasekharan and U.-J. Wiese, Nucl. Phys. B492 (1997) 455.

[21] M. Hermele, M. P. A. Fisher, and L. Balents, Phys. Rev. B69 (2004) 064404.

[22] N. Shannon, G. Misguich, and K. Penc, Phys. Rev. B69 (2004) 220403.

[23] R. Brower, S. Chandrasekharan, and U.-J. Wiese, Phys. Rev. D60 (1999) 094502.

[24] R. Brower, S. Chandrasekharan, S. Riederer, and U.-J. Wiese, Nucl. Phys. B693 (2004) 149.

[25] U.-J. Wiese, Annalen der Physik 525 (2013) 777.

[26] H. P. Büchler, M. Hermele, S. D. Huber, M. P. A. Fisher, and P. Zoller, Phys. Rev. Lett. 95 (2005) 040402.

[27] M. Müller, I. Lesanovsky, H. Weimer, H. P. Büchler, and P. Zoller, Phys. Rev. Lett. 102 (2009) 170502;

[28] M. Müller, I. Lesanovsky, H. Weimer, H. P. Büchler, and P. Zoller, Nature Physics 6 (2010) 382.

[29] E. Zohar, J. Cirac, B. Reznik, Phys. Rev. Lett. 109 (2012) 125302.

[30] D. Banerjee, M. Dalmonte, M. Müller, E. Rico, P. Stebler, U.-J. Wiese, and P. Zoller, Phys. Rev. Lett. 109 (2012) 175302.

[31] L. Tagliacozzo, A. Celi, A. Zamora, and M. Lewenstein, Ann. Phys. 330 (2013) 160.

[32] L. Tagliacozzo, A. Celi, P. Orland, and M. Lewenstein, Nature Communications 4 (2013) 2615.

[33] D. Banerjee, M. Bögli, M. Dalmonte, E. Rico, P. Stebler, U.-J. Wiese, and P. Zoller, Phys. Rev. Lett. 110 (2013) 125303.

[34] D. Banerjee, F.-J. Jiang, P. Widmer, and U.-J. Wiese, JSTAT (2013) P12010.

[35] A. Vishwanath, L. Balents, and T. Senthil, Phys. Rev. B69 (2004) 224416.

[36] T. Sentil, A. Vishwanath, L. Balents, S. Sachdev, and M. P. A. Fisher, Science 303 (2004) 1490.

[37] T. Sentil, L. Balents, S. Sachdev, A. Vishwanath, and M. P. A. Fisher, Phys. Rev. B70 (2004) 144407.

[38] G. 't Hooft, Nuci. Phys. B153 (1979) 141.

[39] J. Liu, A. Sandvik, and L. Balents, Phys. Rev. Lett. 99 (2007) 207203. 